Sādhanā Vol. 28, Part 6, December 2003, pp. 1019-1026. C Printed in India

\title{
A third-order active-R filter with feedforward input signal
}

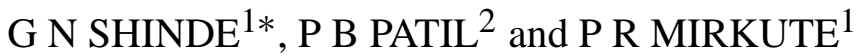 \\ ${ }^{1}$ Department of Electronics, Yeshwant College, Nanded 431 602, India \\ ${ }^{2}$ Department of Physics, Dr B A M University, Aurangabad 431 001, India \\ e-mail: shindegn@yahoo.co.in
}

MS received 9 July 2001; revised 8 September 2003

\begin{abstract}
A realization of voltage-mode transfer functions with feedforward input signal for third-order active-R filter using an oprational amplifier has been presented. This filter is useful for high frequency operation, monolithic IC implementation and is easy to design. The single circuit gives three filter functions, low pass, high pass and band pass. This filter circuit can be used for different $Q$ and $f_{0}$ with high passband gain. This gives better stop band attenuation and sharper cut-off at the edge of the passband.
\end{abstract}

Keywords. Filters; third-order; active-R; feedforward; passband.

\section{Introduction}

The operational amplifier (op. amp.) is now accepted as the basic active component for an inductorless filter. The circuit is realised using single pole (as "integrator") behaviour of an internally compensated operational amplifier (Huelsman 1971; Sun 1983; Mohan \& Patil 1992; Shinde \& Patil 2002). The filter without the capacitor is called an active-R filter; and has received much attention due to its potential advantages in terms of miniaturization, ease of design and high frequency performance (Srinivasan 1992; Kadam \& Mahajan 1995; Shinde \& Mirkute 2003). It has been also pointed out in the literature that active-R networks offer substantially low $\omega_{0}$ sensitivity characteristics as compared to R-C active structures (Soderstand \& Mitra 1971). Although several papers have been published on evaluation of the second order active-R filter, we have not came across any reported circuit regarding direct coupled circuit for realizing third-order active-R filters with feedforward input signals. This gives greater stop-band attenuation and sharper cut-off at the edge of the passband.

This paper proposes realization and design method for third-order active- $\mathrm{R}$ filter with feedforwarded input signal. This filter circuit gives three filter functions, low pass, high pass and band pass with better gain roll-off and passband gain. The circuit is designed and studied for different values of circuit merit factor $Q$ and centre frequency $f_{o}$. The theory shows that for practical realization, the $Q$ has a lower limit $(Q \geq 0 \cdot 5)$ as $f_{o}$ is assumed. Although this circuit works in the low frequency range due to laboratory limitations, the idea can be used for implementation of the circuit at microwave frequency range as operational amplifiers are used in the comparator mode.

\footnotetext{
${ }^{*}$ For correspondence
} 


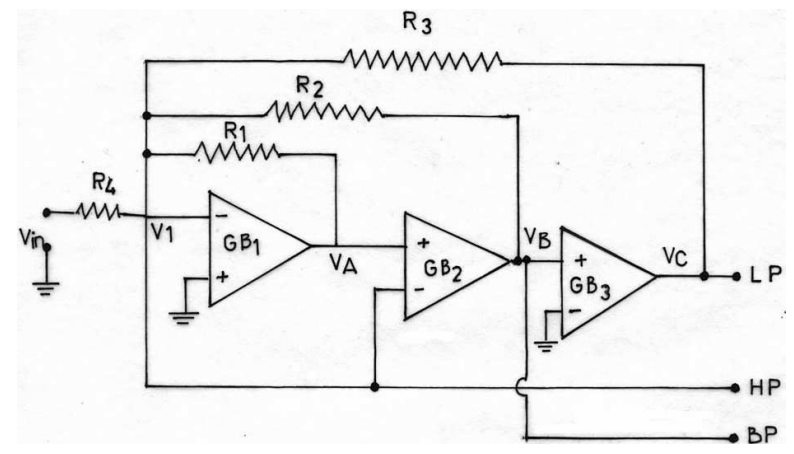

Figure 1. Circuit diagram for active$\mathrm{R}$ third-order filter with feedforword input signal.

\section{Circuit configuration}

The proposed active- $\mathrm{R}$ third-order filter circuit diagram is shown in figure 1 . With the advent of the high frequency roll-off in the response of the op.amp, the circuit is constructed with multiple feedback. There are three op.amplifiers ( $\mu$ A 741), with identical gain bandwidth product as an active element, and four resistances. This filter gives multiple outputs, which tend three filter functions, low pass, high pass and band pass. The feedback is introduced through resistances $R_{1}, R_{2}, R_{3}$ from the output of the three op.amplifiers to inverting input of the first op.amp. The feedforwarded signal is given by connecting the inverting input of the first op.amp. to the inverting input of the second op.amp.

\section{Circuit analysis and design equations}

The single-pole model of an op.amp. leads to complex gain and the transfer function is given by (Srinivasan 1992)

$$
A(s)=A_{0} \omega_{0} /\left(S+\omega_{0}\right),
$$

where $A_{o}=$ open loop d.c. gain, $\omega_{0}=$ open loop $3 \mathrm{~dB}$ bandwidth, $G B=A_{0} \omega_{0}=$ gain bandwidth product of op.amp.

$$
A(s)=A_{0} \omega_{0} / S=G B / S, \text { where } S \gg \omega_{0} .
$$

This shows that the op. amp. is an "integrator",

Thus the active-R third-order filter transfer function at three different terminals are given below.

The voltage transfer function for low pass filter

$$
T_{L P}(S)=\frac{-\left(1 / R_{4}\right) G B_{1} G B_{2} G B_{3}}{X_{1} S^{3}+X_{2} S^{2}+X_{3} S+X_{4}} .
$$

The voltage transfer function for band pass filter

$$
T_{B P}(S)=\frac{-\left(1 / R_{4}\right) G B_{1} G B_{2} S}{X_{1} S^{3}+X_{2} S^{2}+X_{3} S+X_{4}} .
$$


The voltage transfer function for high pass filter

$$
T_{H P}(S)=\frac{\left(1 / R_{4}\right) S^{3}}{X_{1} S^{3}+X_{2} S^{2}+X_{3} S+X_{4}},
$$

where

$$
\begin{aligned}
& X_{1}=\left[\left(1 / R_{1}\right)+\left(1 / R_{2}\right)+\left(1 / R_{3}\right)+\left(1 / R_{4}\right)\right], X_{2}=\left[\left(G B_{1} / R_{1}\right)-\left(G B_{2} / R_{2}\right)\right], \\
& \left.X_{3}=\left[\left(G B_{1} G B_{2} / R_{2}\right)-\left(G B_{2} G B_{3} / R_{3}\right)\right], X_{4}=\left[\left(G B_{1} G B_{2} G B_{3}\right) / R_{3}\right)\right]
\end{aligned}
$$

The circuit was designed using coefficient matching technique with general third-order filter transfer function (Mohan N \& Patil 1992; Shinde 2002).

$$
T(S)=\frac{H_{3} S^{3}+H_{2} S^{2}+H S+H_{0}}{S^{3}+S^{2} \omega_{0}[(1 / Q)+1]+S \omega_{0}^{2}[(1 / Q)+1]+\omega_{0}^{3}}
$$

By comparing (3),(4), and (5) with (6), we get the design equation as

$$
\begin{aligned}
\omega_{0}^{3} & =\left[G B_{1} G B_{2} G B_{3}\right] / R_{3}, \\
\omega_{0}^{2}[(1 / Q)+1] & =\left[\left(G B_{1} G B_{2} / R_{2}\right)-\left(G B_{2} G B_{3} / R_{3}\right)\right], \\
\omega_{0}[(1 / Q)+1] & =\left[\left(G B_{1} / R_{1}\right)-\left(G B_{2} / R_{2}\right)\right], \\
1 & =\left(1 / R_{1}\right)+\left(1 / R_{2}\right)+\left(1 / R_{3}\right)+\left(1 / R_{4}\right) .
\end{aligned}
$$

Values of $R_{1}, R_{2}, R_{3}$, and $R_{4}$ can be calculated using these equations for different values $Q$ and $f_{0}$ (table 1 ). All values of resistances are impedance scale by 10 for practical realization of this filter.

\section{Sensitivity}

\begin{tabular}{|c|c|c|c|c|c|c|c|c|}
\hline \multirow[b]{2}{*}{$Q$} & \multicolumn{4}{|c|}{ Designed value (ohms) } & \multicolumn{4}{|c|}{ Experimental value (ohms) } \\
\hline & $R_{1}$ & $R_{2}$ & $R_{3}$ & $R_{4}$ & $R_{1}$ & $R_{2}$ & $R_{3}$ & $R_{4}$ \\
\hline $0 \cdot 1$ & 50 & $2 \cdot 850 \mathrm{~K}$ & $1.7 \mathrm{M}$ & 12 & 50 & $2 \cdot 8 \mathrm{~K}$ & $1.7 \mathrm{M}$ & 10 \\
\hline 0.5 & 186 & $10.453 \mathrm{~K}$ & $1.75 \mathrm{M}$ & 10 & 180 & $10 \cdot 4 \mathrm{~K}$ & $1.7 \mathrm{M}$ & 10 \\
\hline 1 & 280 & $15 \cdot 68 \mathrm{~K}$ & $1.75 \mathrm{M}$ & 10 & 280 & $15 \cdot 6 \mathrm{~K}$ & $1.7 \mathrm{M}$ & 10 \\
\hline 10 & 509 & $28.50 \mathrm{~K}$ & $1.75 \mathrm{M}$ & 10 & 500 & $28 \mathrm{~K}$ & $1.7 \mathrm{M}$ & 10 \\
\hline
\end{tabular}

The sensitivities of $Q$ and $\omega_{0}$ in this active-R third-order filter to both passive and active values are as follows.

(a) $S_{R_{1}}^{\omega_{0}}=-\left(\omega_{0} / 3 G B_{1}\right)[(1 / Q)+1]$.

(b) $S_{R_{2}}^{\omega_{0}}=\left(\omega_{0}^{2} / 3 G B_{1} G B_{2}\right)[(1 / Q)+1]$.

(c) $S_{R_{3}}^{\omega_{0}}=\left(\omega_{0}^{2} / 3\right)\left[\left[\omega_{0}^{3} /\left(G B_{1}, G B_{2}, G B_{3}\right)\right]-1\right]$.

(d) $S_{R_{4}}^{\omega_{0}}=\left(1 / 3 R_{4}\right)$.

Table 1. Resistance values for some values of $Q$. 
(e) $S_{G B_{1}}^{\omega_{0}}=(1 / 3)$.

(f) $S_{G B_{2}}^{\omega_{0}}=(1 / 3)$.

(g) $S_{G B_{3}}^{\omega_{0}}=(1 / 3)$

(h) $S_{R_{1}}^{Q}=\left[1 /\left(3 Q^{2} R_{1}\right)\right]\left[\left(3 G B / \omega_{0}[(1 / Q)+1]\right)+1\right]$.

(i) $S_{R_{2}}^{Q}=\left[1 /\left(3 Q^{2} R_{2}\right)\right]\left[\left(3 G B / \omega_{0}[(1 / Q)+1]\right)+1\right]$.

(j) $S_{R_{3}}^{Q}=\left(1 / 3 Q^{2}\right)\left[\left(\omega_{0}^{3} / G B_{1} G B_{2} G B_{3}\right)-1\right]$.

(k) $S_{R_{4}}^{Q}=\left(1 / 3 Q^{2} R_{4}\right)$.

(l) $S_{G B_{1}}^{Q}=\left(1 / 3 Q^{2}\right)\left[1-\left[3 G B_{1} / R_{1} \omega_{0}[(1 / Q)+1]\right]\right]$.

(m) $S_{G B_{2}}^{Q}=\left(1 / 3 Q^{2}\right)\left[1+\left[3 G B_{2} / R_{2} \omega_{0}[(1 / Q)+1]\right]\right]$.

(n) $S_{G B_{3}}^{Q}=\left(G B_{2}\right) / 3 Q^{2}$.

Thus, passive and active sensitivities are all less than unity. So for all practical purposes this circuit is stable as these sensitivities are very low (Soderstand \& Mitra 1971; Shinde 2002).

\section{Experimental}

The circuit performance was studied with different values of $Q(Q=0 \cdot 02,0 \cdot 04,0 \cdot 06,0 \cdot 08$, $0 \cdot 1,0.5$ and 10 with constant $f_{0}=10 \mathrm{kHz}$ ). The table 1 shows resistance values of same different $Q$ for $G B=2 \pi(800) \mathrm{rad} / \mathrm{sec}$ and $f_{0}=10 \mathrm{kHz}$. Same circuit was also studied for different values of designed centre frequency $f_{0}\left(f_{0}=10 \mathrm{kHz}\right.$ and $100 \mathrm{kHz}$ with constant $Q=10$ ). The observed frequency response shows good agreement with theoretical results. The general range of these frequency response for this active - $\mathrm{R}$ filter is from $10 \mathrm{~Hz}$ to $1 \mathrm{MHz}$ as operating range of this op.amp. is $10 \mathrm{~Hz}$ to $1.2 \mathrm{MHz}$. Following observations are noticed from experimental study at three different terminals; low pass, high pass and band pass filter function for different $Q$ and $f_{o}$.

\section{Result and discussion}

\subsection{Low pass response}

Low pass(LP) responses for different values of $Q$ are shown in figure 2 . The observed centre frequency is also in good agreement with the designed value. It is noticed that this circuit has high passband gain and that the gain roll-off is about $54 \mathrm{~dB} / \mathrm{decade}$. It is observed that the below $10 \mathrm{kHz}$ for higher $Q(Q \geq 10)$, the responses are independent of $Q$.

The responses for different values of are $f_{0}$ shown in figure 3 . As the limitation of op.amp. gain and limitation of input signal source has to be very low, we have not studied the filter response for $f_{o}=1 \mathrm{kHz}$. The theoretical result shows that the gain is very high, about $164 \mathrm{~dB}$ in passband. Also for practical realization, resistances $R_{1}, R_{2}, R_{3}$ and $R_{4}$ must be positive. Hence there is a lower limit on $f_{o}$ if $Q$ is assumed or vice versa. The response shows moderate psssband gain for $f_{0} \geq 10 \mathrm{kHz}$ without disturbing the designed centre frequency. There is no overshoot any where in the response. It is also noticed that this filter response for $f_{0}=100 \mathrm{kHz}$ have passband gain $(42 \mathrm{~dB})$ with $60 \mathrm{~dB}$ gain roll-off. Many reported circuits show very low passband gain for $f_{0}=100 \mathrm{kHz}$ (Kadam \& Mahajan 1995). 


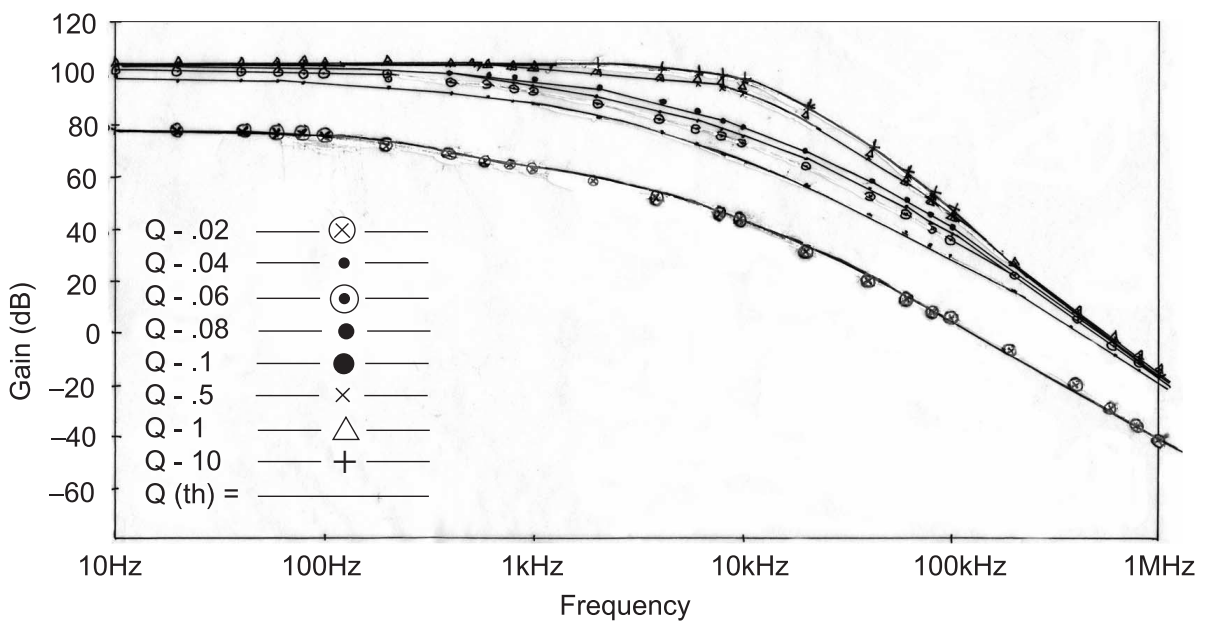

Figure 2. Low pass response for different $Q$.

\subsection{Band pass response}

The band pass (BP) response for different $Q$ is shown in figure 4 . The passband gain decreases with decreasing $Q$ value. Also, the bandwidth decreases with increasing $Q$. It is observed that the designed centre frequency is shifted for lower values of $Q$. Thus this filter is useful for $Q \geq 0 \cdot 5$.

Figure 5 shows bandpass response for different $f_{0}$. It is noticed that very high passband gain appears in the response. It is observed that passband gain decreases and bandwidth increases with increase in centre frequency. However, the circuit shows some degradation for lower frequency range gain roll-off. Thus one has to select higher values of $Q(Q \geq 10)$.

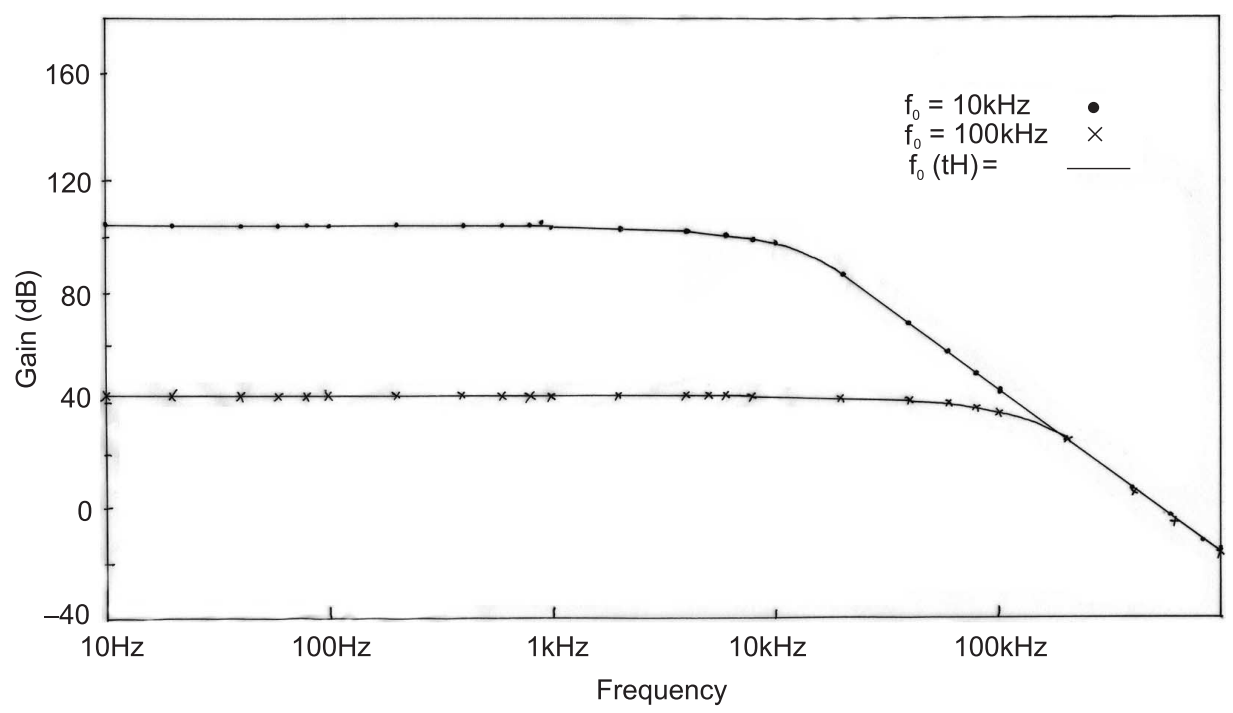

Figure 3. Low pass response for different $f_{0}$. 


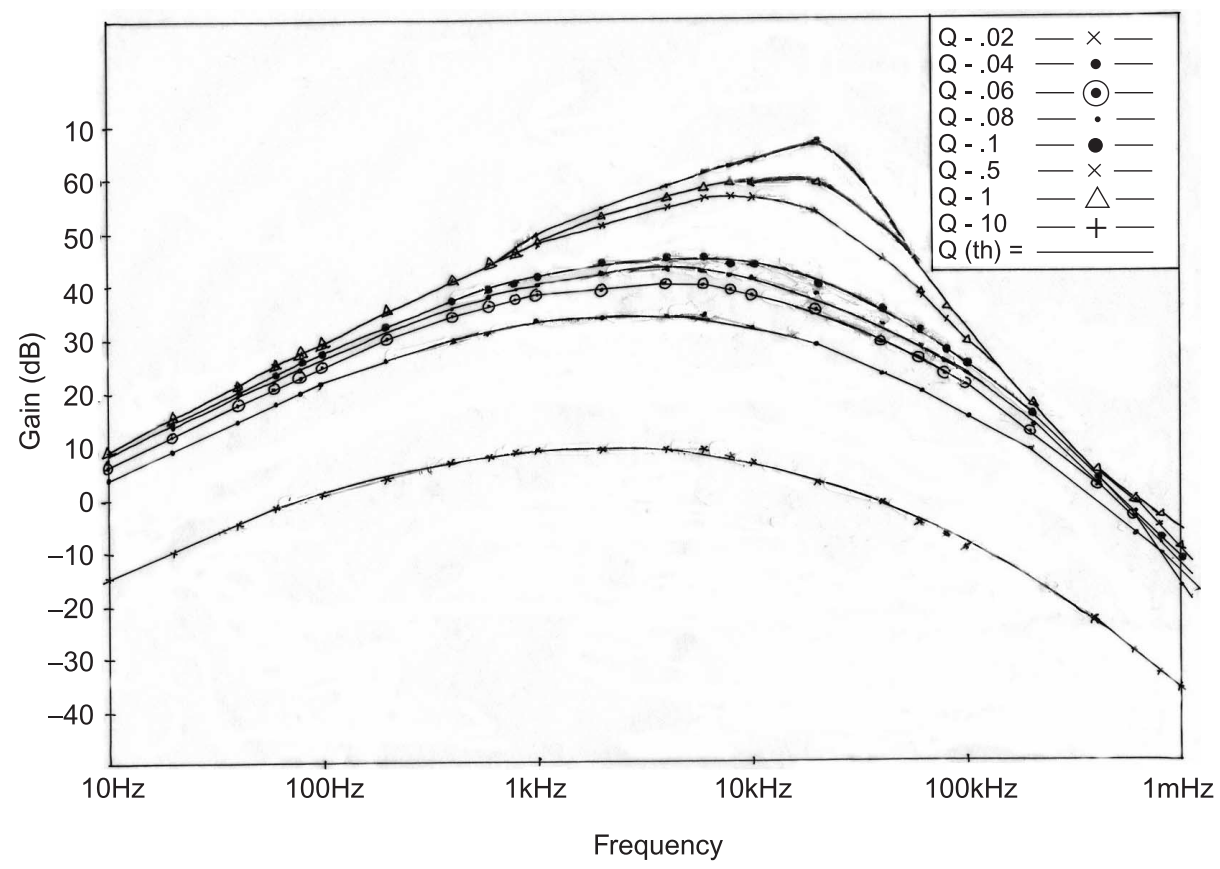

Figure 4. Band pass response for different $Q$

\subsection{High pass response}

High pass (HP) responses for different values of $Q$ are shown in figure 6 . The response is extended up to $500 \mathrm{~Hz}$ at lower frequencies, as many reported circuits start their response from $1 \mathrm{kHz}$ (Hioashimura 1992). The response shows undisturbed designed centre frequency

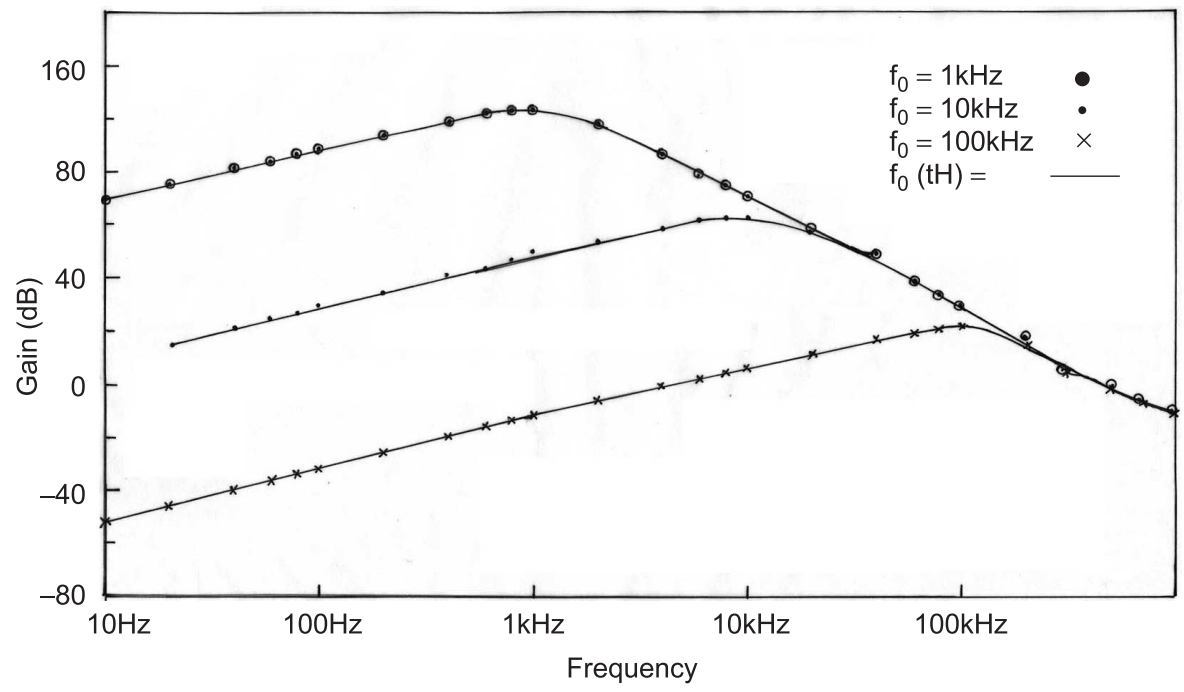

Figure 5. Band pass response for different $f_{0}$ 


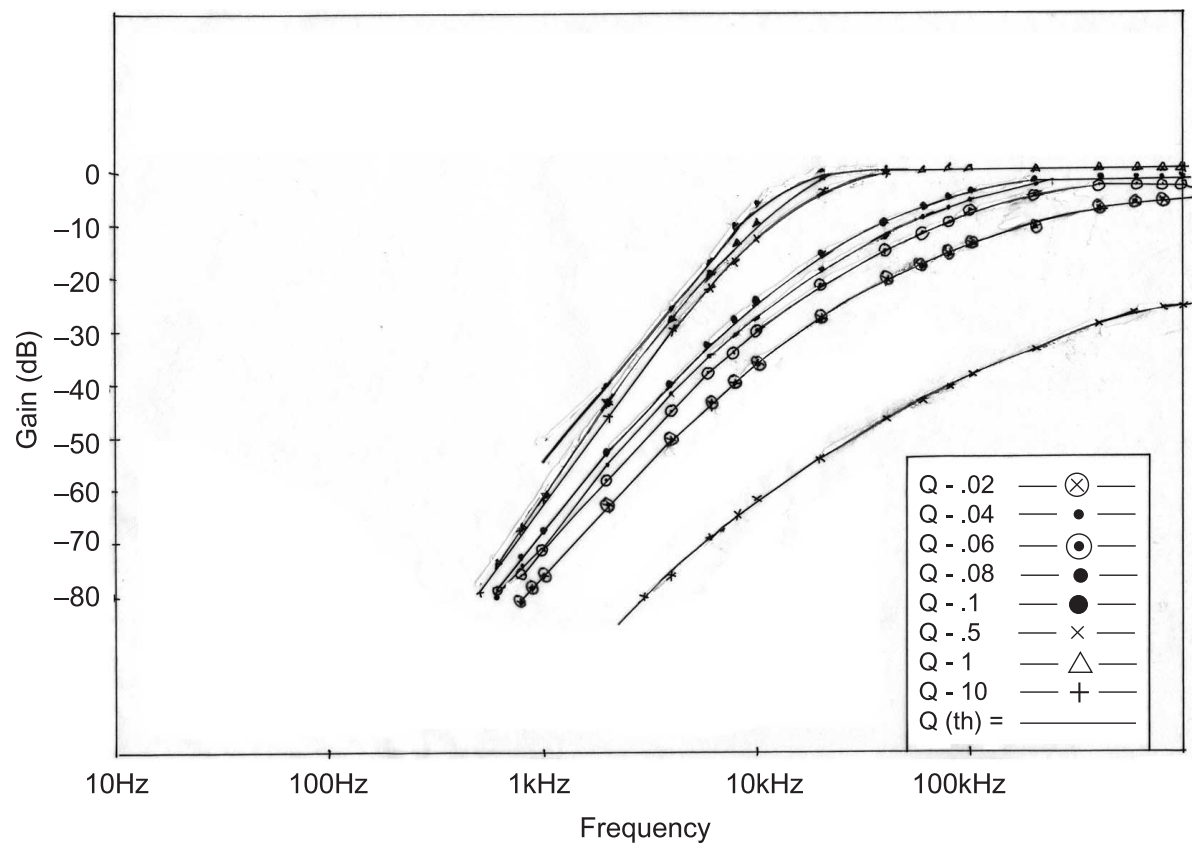

Figure 6. High pass response for different $Q$.

for $Q \geq 0.5$ without any overshoot and gain roll-off was of $54 \mathrm{~dB} / \mathrm{decade}$. It is also observed that pass band gain decreases with decrease in $Q$ and the lowest passband gain of $-26 \mathrm{~dB}$ is obtained for $Q=0.02$.

Figure 7 shows high pass response for different designed centre frequencies. Designed centre frequency is not disturbed with gain roll-off of about $54 \mathrm{~dB} /$ decade. This response has better passband gain $(0 \mathrm{~dB})$, which is independent of centre frequency. The response at lower

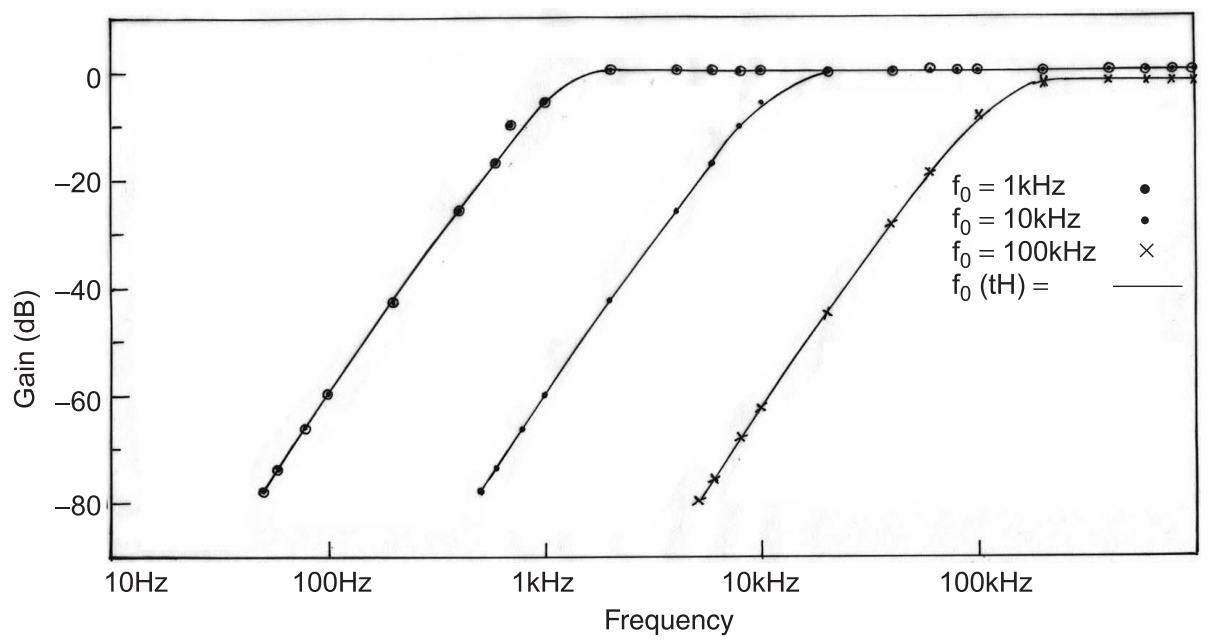

Figure 7. High pass response for different $f_{0}$. 
frequency is extended up to $50 \mathrm{~Hz}$ for $f_{o}=1 \mathrm{kHz}$. This filter circuit can be used for any value of centre frequency for higher $Q$ without any overshoot.

\section{Conclusion}

A realization of voltage - mode transfer function for a third-order active- $\mathrm{R}$ filter using op.amp. with feedforword input signal has been presented. Since this circuit is composed only of op.amplifiers and four resistances, it is suitable for high frequency operation and monolithic IC implementation. Also, this single filter circuit gives three filter functions; low pass, band pass and high pass for high passband gain and high value of $Q$. This filter gives better stopband attenuation and sharper cut-off at the edge of the passband, and can be used for different values of $Q$ and $f_{0}$.

The authors would like to acknowledge the University Grants Commission (Western Regional Office), Pune for financial support for part of the experimental work.

\section{References}

Hioashimura M 1992 Active-R realization of current mode high pass filter. Int. J. Electron. 73: 1279_ 1283

Huelsman L P 1971 Equal-valued capacitor active-RC network realisation of a 3rd order low pass Butterworth characteristics. Electron. Lett. 7: 291-293

Kadam A B, Mahajan A M 1995 Effect of positive feedback on the response of active-R filter. $J$. Instrum. Soc. India 25: 48-55

Mohan N, Patil R L Ripple pass function and their active-R realization. Indian J. Pure Appl. Phys. 30: $749-750$

Shinde G N, Patil P B 2002 Study of active-R second-order filter using feedback at non-inverting terminals. Bull. Pure Appl. Sci. D21: 23-31

Shinde G N, Mirkute P R, Achole P D 2003 Second order active-R filter with multiple feedback for different Q. Indian J. Phys. B77: 72-80

Soderstand M A, Mitra S K 1971 Sensitivity analysis of third-order filter. Int. J. Electron. 30: 265-273

Srinivasan S 1992 Synthesis of transfer function using the operation amplifier pole. Int. J. Electron. 73: $1279-1283$

Sun Zhi-Xiao 1983 Active-R filter: a new biquadratic with four terminals. Int. J. Electron. 54: 523-530 